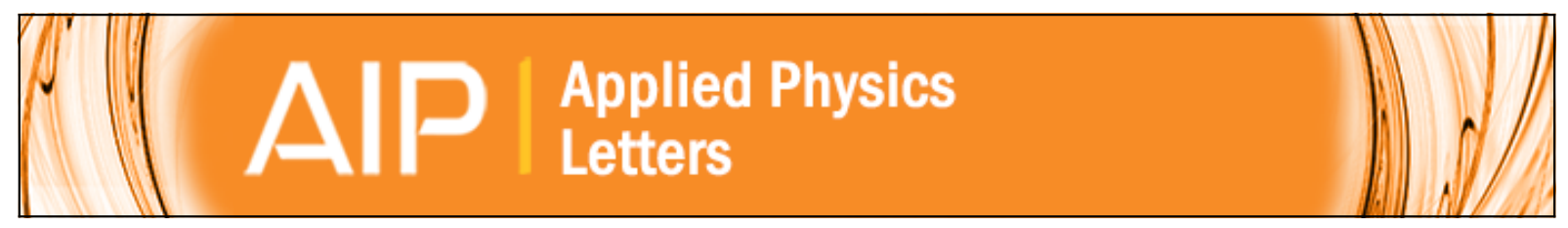

\title{
High performance Feussner-type polarizers based on stretched poly(ethylene- terephthalate) films
}

J. C. Martnez-Antón and E. Bernabeu

Citation: Applied Physics Letters 80, 1692 (2002); doi: 10.1063/1.1457529

View online: http://dx.doi.org/10.1063/1.1457529

View Table of Contents: http://scitation.aip.org/content/aip/journal/apl/80/10?ver=pdfcov

Published by the AIP Publishing

\section{Articles you may be interested in}

Polarization-dependent optical characterization of poly(phenylquinoxaline) thin films

J. Appl. Phys. 100, 063106 (2006); 10.1063/1.2349471

Interferometric determination of the anisotropic refractive index dispersion of poly-( $p$-phenylene-vinylene) Appl. Phys. Lett. 86, 201119 (2005); 10.1063/1.1935035

High extinction ratio antiresonant reflecting optical waveguide-type polarizers with large core diameter Appl. Phys. Lett. 86, 071114 (2005); 10.1063/1.1865336

Molecular weight dependence of birefringence of thin films of the conjugated polymer poly[2-methoxy-5- (2 ethyl-hexyloxy)-1, 4-phenylenevinylene]

Appl. Phys. Lett. 84, 3792 (2004); 10.1063/1.1739513

Switchable optical polarizer based on electrochromism in stretch-aligned polyaniline Appl. Phys. Lett. 83, 1307 (2003); 10.1063/1.1602556

\section{NEW! Asylum Research MFP-3D Infinity ${ }^{\text {TM }}$ AFM} Unmatched Performance, Versatility and Support

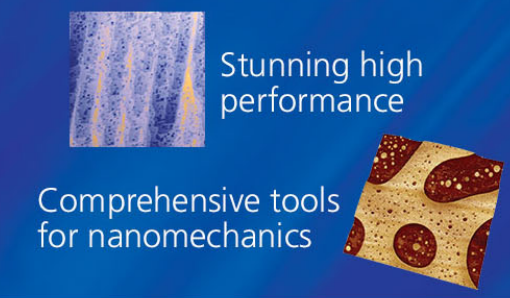

Simpler than ever to GetStarted ${ }^{\mathrm{T}}$

Widest range of accessories

for materials science and bioscience

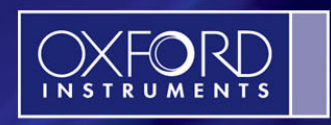

The Business of Science

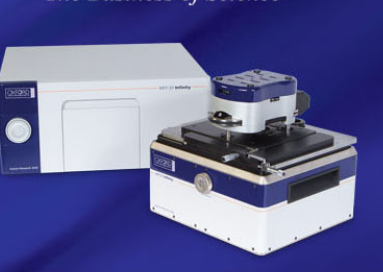




\title{
High performance Feussner-type polarizers based on stretched poly(ethylene-terephthalate) films
}

\author{
J. C. Martínez-Antón ${ }^{\text {a) }}$ and E. Bernabeu \\ Departamento de Optica, Facultad de Físicas, Universidad Complutense de Madrid, 28040 Madrid, Spain
}

(Received 31 July 2001; accepted for publication 4 January 2002)

\begin{abstract}
Stretched poly(ethylene-terephthalate) films appear to be an interesting optical plastic for use in polarization control devices. By means of stretching ratios its birefringence, in practice, can be tailored from $\sim 0$ to $\sim 0.17$. It has a very wide transmission window $(\sim 0.32-5.70 \mu \mathrm{m})$ and good thermal, mechanical, and chemical properties. We propose using it for polarizers based on the Feussner design. By implementing this configuration with a biaxially stretched film, we have obtained a polarizer with an achromatic extinction ratio better than 1 part in 50000 . This is comparable with conventional Glan-Thompson polarizers but with additional advantages. The principal refractive indices of the film (in the 0.43-5.7 $\mu \mathrm{m}$ range) and the operational range of the polarizer presented are also provided. (C) 2002 American Institute of Physics.
\end{abstract}

[DOI: $10.1063 / 1.1457529]$

Many types of polarizer have been reported in the literature. ${ }^{1}$ They are based on several physical mechanisms, basically (1) the selective absorption of one polarization component in dichroic materials, (2) the polarization induced in reflected beams near the Brewster angle, and (3) the different reflection, refraction, or scattering in birefringent materials depending on the polarization. Multilayer stacks are also used to produce interferential polarizers, which basically enhance the previous polarization mechanisms for limited spectral bands. ${ }^{2,3}$ Conventional prism polarizers belong to the third category, in that the Feussner-type design ${ }^{1}$ consists of an anisotropic layer inserted between two isotropic media (see Fig. 1). In this configuration the polarization components $(P$ and $S)$ encounter different refractive indices at the prism-film interface. By appropriately choosing the angle of incidence and the refractive index of the prism couple, one of the polarization components undergoes total reflection, and the other propagates out. As pointed out by Bennett ${ }^{1}$ this design has several advantages: (1) the imaging through the polarizer is anastigmatic, (2) large field angles or prisms with compact sizes can be obtained, and (3) the birefringent material is used economically. An optimum configuration is achieved when the main optical axis of the birefringent material is oriented perpendicular to the film surface. This is the case for stretched poly(ethylene-terephthalate) (PET) films which have their axis with the lowest refractive index normal to the surface. Different materials to use with this design have been proposed: sodium nitrate Refs. 4-6, among others, mica, ${ }^{7}$ etc. However, although the Feussner design shows interesting advantages, it has not had widespread commercial use. Difficulties in producing appropriate films, low environmental resistance, or poor birefringence may somewhat explain why.

PET becomes highly anisotropic when stretched (see its molecular structure in Fig. 2). A maximum in-plane/out-ofplane birefringence of $n_{x}-n_{z} \sim 0.19$ has been found

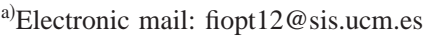

experimentally. ${ }^{8}$ A biaxial stretching process leads to a biaxial optical medium with axes oriented like they are in Fig. 1. Depending on the stretching ratio, the optical properties of PET can be advantageously tailored. ${ }^{8}$

For a proper polarizer design at any wavelength interval, we require knowledge of the refractive indices of the film inserted. We used and characterized a PET film obtained from Goodfellow Cambridge Ltd., ${ }^{9}$ that had a nominal thick-
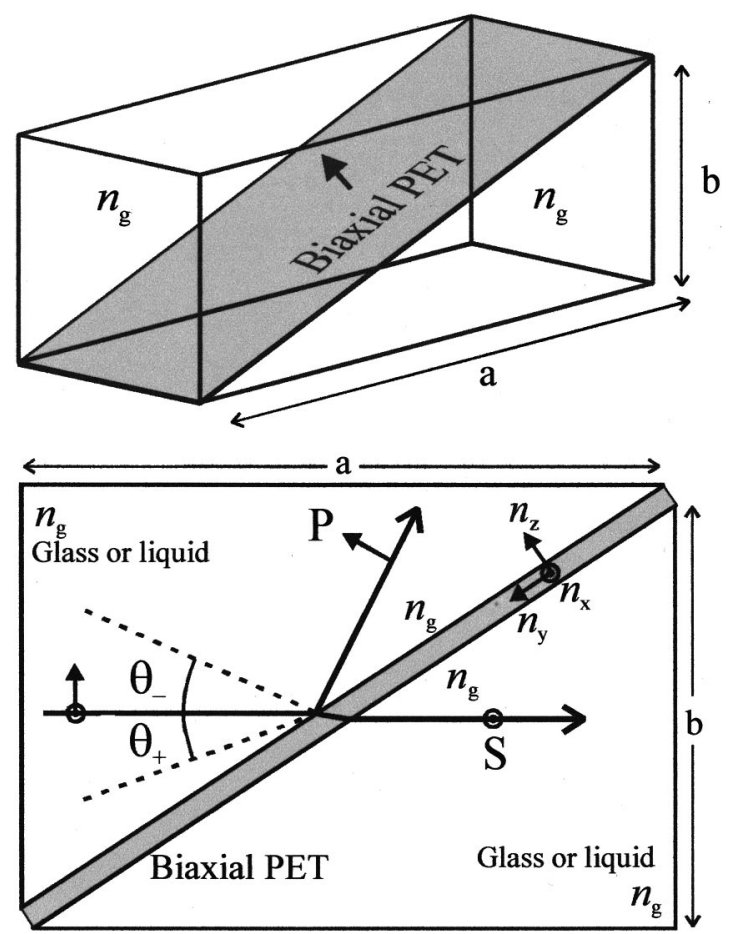

FIG. 1. Feussner-type prism polarizer. An anisotropic film is inserted between two isotropic prisms. We consider the optical axes as the $Z$ axis $\left(n_{z}\right)$ oriented normal to the surface of the film, and the $X$ axis $\left(n_{x}\right)$ [or $Y$ axis $\left.\left(n_{y}\right)\right]$ oriented along the surface of the film and perpendicular (parallel) to the plane of incidence. We have total reflection of the $P$ component at an appropriate angle of incidence when $n_{g}>n_{z}$. Internal acceptance angles are expressed by $\theta_{+}$and $\theta_{-}$which correspond to the critical angles for $P$ and $S$ polarization, respectively. 
<smiles>CCCOC(=O)c1ccc(C(=O)OCCOC(=O)c2ccc(C(=O)OC)cc2)cc1</smiles>

FIG. 2. Molecular structure of poly(ethylene-terephthalate).

ness of $d=23 \mu \mathrm{m}$, and was biaxially stretched (3:4 ratio) in sequential mode. The principal refractive indices, $n_{x}, n_{y}$, and $n_{z}$, were obtained in the visible and IR by different methods. ${ }^{10,11}$

We measured the transmission (reflection) of the film, as a function of the wavelength $(\lambda)$, for different incidence angle $(\phi),{ }^{10,11}$ or as a function of the incidence angle for different wavelengths. The sample was oriented in order to have the neutral axes parallel and perpendicular to the plane of incidence. In the absence of absorption, localization of the interference maxima and minima [Fig. 3, curve (B), in the visible range] allows the determination of $n_{x}, n_{y}$, and $n_{z}$. For instance, with $S$ polarization the extremes obey the interference condition:

$$
\begin{aligned}
n^{2}(\lambda)= & n_{0}^{2} \sin (\phi)^{2}+(\lambda / 4 d)^{2}\left(N_{0}-i\right)^{2}, \\
& \text { with } i=0,1,2,3, \ldots .
\end{aligned}
$$

Equation (1) is applicable for a wavelength scan and then $\lambda=\lambda_{i}$ and $\phi$ is fixed, or for an angular scan and then $\phi$ $=\phi_{i}$ and $\lambda$ is fixed. $N_{0}$ is the reference interference order that corresponds to the lowest wavelength extreme or the lowest angular extreme, respectively. By relating several measurements we can extract $N_{0}, n_{x}$, or $n_{y}$, and $d$. Finally, when using incident $P$ polarization and considering now that the $X$ axis is in the plane of incidence, we have $\mathrm{e}^{10,11}$

$$
n_{z}^{2}(\lambda)=\frac{n_{0}^{2} \sin ^{2}(\phi)}{\left[1-\left(\lambda / 4 n_{x} d\right)^{2}\left(N_{0}-i\right)^{2}\right]},
$$

from which we can obtain $n_{z}$.

For the visible range measured $(0.43-0.8 \mu \mathrm{m})$, PET is nonabsorbing and determination of interference extremes is simple. ${ }^{10}$ However, in the mid-IR, the presence of absorption bands hinders a correct determination of interference ex-

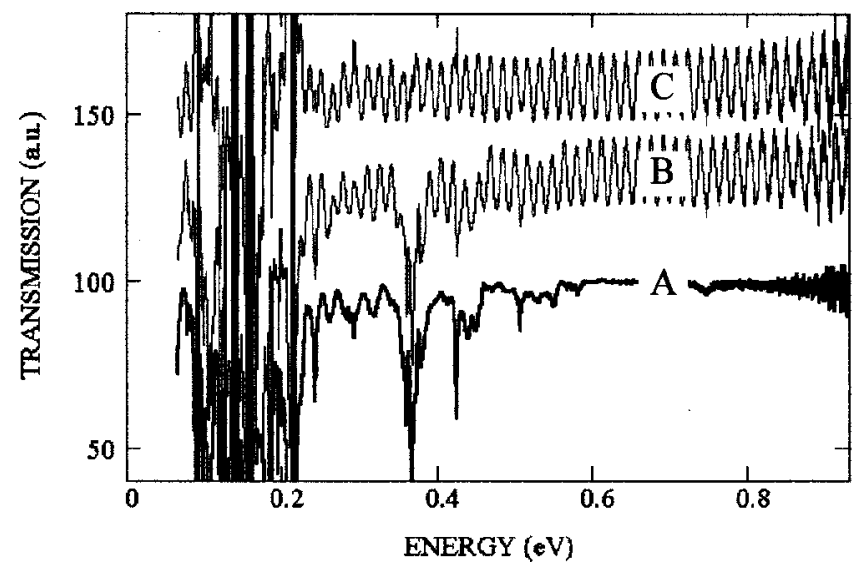

FIG. 3. Curve (A). Transmittance of the polyester film measured at $60^{\circ}$ and $P$ polarization (close to the Brewster angle). The interference reflection effects are negligible but absorption bands remain. Curve (B): Transmission curve obtained at $75^{\circ}$ (both interference and absorption are present). Curve (C) Same as (B) but with the absorption removed using the data of curve (A).

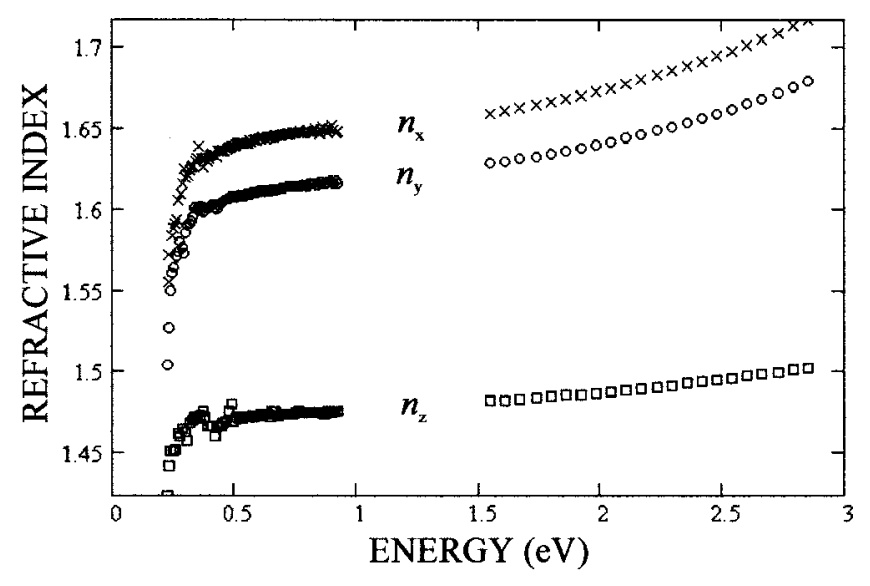

FIG. 4. Refractive indices of the PET film (thickness of $23 \mu \mathrm{m}$ ). The axes are oriented the same way as in Fig. 1.

tremes (see Fig. 3). The approach we have chosen consists of measuring at the Brewster angle. Then, under $P$-polarized light, there is no interference modulation, i.e., only absorption affects the transmittance. From this measurement, we can obtain the internal transmittance and compensate for the effect of absorption bands in other transmission spectra, leaving only interference-alone modulation (see Fig. 3). The principal refractive indices thereby obtained are shown in Fig. 4.

We made a polarizer prototype consisting of two isotropic equilateral prisms (SF18 glass, $n_{d}=1.721$ ) and the above mentioned film inserted in between. A mixture of bromonaphthalene and iodonaphthalene $(n=1.660)$ is used as the index matching liquid between the prisms and the film. This mixture is PET compatible. ${ }^{12}$ We aligned the $X$ axis according to that in Fig. 1. This precaution is needed because the propagated $S$-polarized beam must be aligned to a neutral axis to maintain a polarization state.

We measured the extinction ratio in a setup that consisted of a collimated beam coming from an incandescent source, next the polarizer to be tested that had an internal angle of incidence of $\phi \sim 63^{\circ}$, a Glan-Thompson analyzer with high polarization purity $\left(\epsilon<10^{-6}\right)$, and a monochomator, followed finally by a detector. The dynamic detection range was increased by using a set of calibrated apertures in order to have a higher signal when the polarizer and the analyzer are crossed. We obtained an achromatic extinction ratio of $\epsilon \sim 2 \times 10^{-5}$ (see Fig. 5) for a circular aperture 15

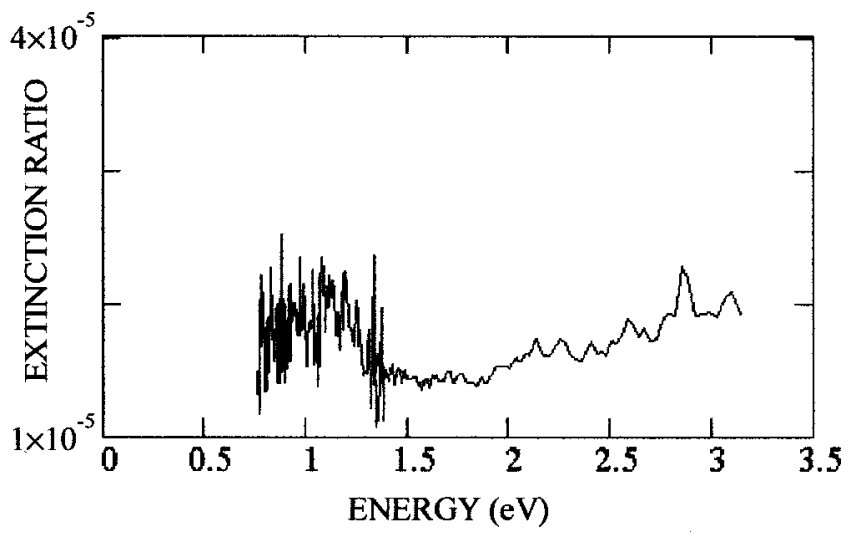




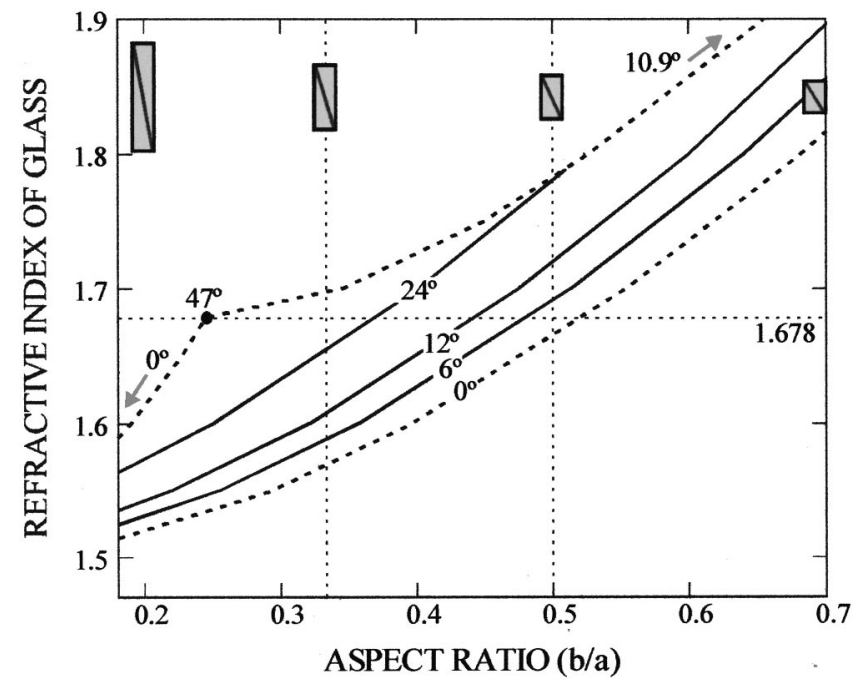

FIG. 6. Operational range of our PET-based polarizer depending on the aspect ratio and refractive index of the surrounding medium. Continuous lines represent constant external apertures $\left(0^{\circ}, 6^{\circ}, 12^{\circ}\right.$, and $\left.24^{\circ}\right)$, that are considered to be symmetrical (i.e., with $\left|\theta_{+}\right|=\left|\theta_{-}\right|$). The area between the $0^{\circ}$ line and the uppermost dashed line permits maximum symmetrical angular apertures. Outside this area and above $m \approx 1^{\prime} 5$ we can operate the polarizer but with an asymmetrical angular aperture. For very high $n_{g}$ we get compact sizes and the aperture tends asymptotically towards $10.9^{\circ}$.

$\mathrm{mm}$ in diameter. Transmittance in the polarization axis is approximately flat with $T \sim 0.83$. This value seems to be due mostly to reflection losses at the entrance and exit faces of the prisms. The polarizer was measured again 4 months after fabrication and we could not find significant variations in its performance.

The external acceptance angle (or angular aperture), associated to $\theta_{+}$and $\theta_{-}$in Fig. 1, can be derived in the following way. According to Fig. 1, the critical angle for $P$ and $S$ polarization components may be expressed, respectively, as

$$
\sin \phi_{P}=\frac{n_{z}}{n_{g}}, \quad \sin \phi_{S}=\frac{n_{x}}{n_{g}},
$$

where $\phi$ is the angle of incidence at the film's interface. The internal angular aperture (i.e., $\left|\theta_{+}\right|+\left|\theta_{-}\right|$) is also $\left|\phi_{S}\right|$ $-\left|\phi_{P}\right|$. The corresponding external angular aperture can be obtained by relating the refraction angles at the entrance and the aspect ratio $(b / a)$. We can estimate the angular operational range for a polarizer based on our particular but representative film. This is shown in Fig. 6. A maximum external and symmetrical aperture of $47^{\circ}$ (i.e., $\pm 23.5^{\circ}$ with respect to the normal of the entrance face) can be obtained for a refractive index match with the surrounding media $\left(n_{g}=n_{x}=1.678\right)$ and an aspect ratio of $b / a=1: 4.08$.

The achievable extinction coefficient is limited by different factors. The most important one seems to be the slight random distribution of the optical axes around a center di- rection. Slight randomization appears both across the surface of the film and in its depth. These "blurred" optical axes introduce a small depolarization in the propagated beam which somewhat degrades the polarization purity. To estimate this effect, a film similar to the one inserted in the polarizer was introduced between crossed polarizers (with $\epsilon<10^{-6}$ ), giving a transmission equivalent of $\epsilon=3.8$ $\times 10^{-5}$, of the same order of magnitude of extinction coefficient as that obtained for our polarizer $\left(2 \times 10^{-5}\right)$. Depolarization due to surface roughness seems to be negligible in our case. On the other hand, crystallization is present inside the films to a different extent, and it depends strongly on the processing conditions. ${ }^{8}$ To avoid depolarization by internal scattering, it is possible to choose commercial crystal clear films in which the amorphous phase is predominant or the crystallites are too small to give significant scattering. Finally, we must be aware of the residual stress of the glass if it is used as the surrounding medium, because this also limits the performance.

In conclusion, we have shown the possibility of fabricating polarizers with performance comparable to that of other classical prism polarizers $\left(\epsilon \sim 10^{-5}-10^{-6}\right)$ but with the additional advantages afforded by the Feussner configuration. Calcite-based classical polarizers work in the $\sim 0.3-2.3 \mu \mathrm{m}$ spectral range, but we may find similar prism polarizers based on yttrium-vanadate $\left(\mathrm{YVO}_{4}\right)$ crystal work in the $0.45-5 \mu \mathrm{m}$ interval or those based on barium borate $(\alpha-$ BBO) crystal, which works from 0.19 to $3.5 \mu \mathrm{m} .{ }^{13}$ PET polarizers could be a cheaper alternative and valid for the 0.32 $5.7 \mu \mathrm{m}$ range. An additional advantage is that PET films are available in practically unlimited sizes, in contrast to crystal optics. PET is UV resistant, it withstands significant temperature variations $\left(-50\right.$ to $\left.+150^{\circ} \mathrm{C}\right)$, and it is chemically quite inert and impermeable.

This work was financially support by the Universidad Complutense Madrid (PR48/01-9883) and by the Comunidad de Madrid (07N/0039/1998), Spain.

${ }^{1}$ J. M. Bennett, Handbook of Optics, edited by M. Bass (McGraw-Hill, New York, 1995), Vol. II, Chap. 3.

${ }^{2}$ J. A. Dobrowolski and A. Waldorf, Appl. Opt. 20, 111 (1981).

${ }^{3}$ I. Hodgkinson and Qi Hong Wu, Appl. Phys. Lett. 74, 1794 (1999).

${ }^{4}$ M. H. de Longchamp, Rev. Opt., Theor. Instrum. 26, 94 (1947).

${ }^{5}$ T. Yamaguti, J. Phys. Soc. Jpn. 10, 219 (1955).

${ }^{6}$ T. Yamaguti, I. Makino, S. Shinoda, and I. Kuroha, J. Phys. Soc. Jpn. 14, 199 (1959).

${ }^{7}$ F. J. Dumont and R. N. Smartt, J. Opt. Soc. Am. 59, 1541 (1970).

${ }^{8}$ M. Cakmak, J. L. White, and J. E. Spruiell, Polym. Eng. Sci. 29, 1534 (1989).

${ }^{9}$ Goodfellow Cambridge Limited, Ermine Business Park, Huntingdon, UK, PE29/PET film Ref. No. ES301191.

${ }^{10}$ J. C. Martínez-Antón, Mater. Lett. (in press).

${ }^{11}$ J. C. Martínez-Antón, J. Opt. Mater (in press).

${ }^{12}$ Cargille Laboratories Inc., 55 Commerce Rd. Cedar Grove, NJ 07009.

${ }^{13}$ Casix, Inc., P.O. Box 1103, Fuzhou, Fujian 350014, People's Republic of China. 